\title{
Fractionation of chlorinated and brominated persistent organic pollutants in several food samples by pyrenyl-silica liquid chromatography prior to GC-MS determination
}

\author{
Belén Gómara ${ }^{\mathrm{a}, \mathrm{b}}$, Carmen García-Ruiz ${ }^{\mathrm{a}}$, María José González ${ }^{\mathrm{b}}$, María Luisa Marina ${ }^{\mathrm{a}, *}$ \\ a Departamento de Química Analítica, Facultad de Química, Universidad de Alcalá, Ctra. Madrid-Barcelona Km. 33.600, \\ 28871 Alcalá de Henares, Madrid, Spain \\ b Departamento de Análisis Instrumental y Química Ambiental, Instituto de Química Orgánica General, CSIC, Juan de la Cierva, 3 28006, Madrid, Spain
}

Received 8 November 2005; received in revised form 21 February 2006; accepted 22 February 2006

\begin{abstract}
A high-performance liquid chromatography (HPLC) method using a column of 2-(1-pyrenyl) ethyldimethylsilylated silica was developed in this work in order to achieve satisfactory and reproducible fractionation of polychlorinated biphenyls (PCBs) from brominated flame retardants (BFRs) (polybrominated diphenylethers, PBDEs; and polybrominated biphenyls, PBBs). After the study of different chromatographic parameters (mobile phase composition and separation temperature were the most important) an isocratic elution with isooctane:toluene (98:2, v/v) at a flow-rate of $1 \mathrm{~mL} / \mathrm{min}$, a temperature of $45^{\circ} \mathrm{C}$, and UV-detection at $225 \mathrm{~nm}$ was selected for fractionation of PCBs (time region, 4.0-5.8 min) from PBDEs (5.8-9.0 min) and from PBBs (5.8-11.0 min). The applicability of this method to food samples was demonstrated by fractionating PCBs from PBDEs in three food samples (cheese, milk, and fish). Interferences from PCBs (present in real samples at much higher concentrations than PBDEs) were removed in this way. In addition, by analysing these samples by gas chromatography-mass spectrometry (GC-MS) with and without previous fractionation we were able to observe an improvement in detection sensitivity for PBDEs after HPLC fractionation.
\end{abstract}

(c) 2006 Elsevier B.V. All rights reserved.

Keywords: Brominated and chlorinated POPs; Fractionation; PYE; HPLC

\section{Introduction}

Polybrominated diphenylethers (PBDEs) and polybrominated biphenyls (PBBs) are two groups of ubiquitous pollutants that are present in environmental samples due to their use as brominated flame retardants (BFRs) to decrease the likelihood and intensity of fire in electronic components, plastics, clothing and a number of other commercial products [1]. There is currently growing interest, particularly in PBDE analysis in environmental and food samples due to the marked increase in the levels of these compounds detected during the last decade [2]. In 2003, the European Community (EC) introduced a new regulation to control the presence of PBDEs in the environment [3], with the intention of introducing new regulations similar to those existing for polychlorodibenzo- $p$-dioxins and poly-

\footnotetext{
* Corresponding author. Tel.: +34 91 8854935; fax: +34 918854971 .

E-mail address: mluisa.marina@uah.es (M.L. Marina).
}

chlorodibenzo furans (PCDD/Fs) and polychlorinated biphenyls (PCBs) for foodstuffs [4] in the near future. These directives involve routine monitoring calling for adaptation of analytical methods in order to provide adequate sensitivity and selectivity so as to allow unambiguous determination of BFR in complex matrices.

In most cases the analytical procedure is based on established methods for similar compounds, such as PCBs and PCDD/Fs. The final clean-up step of these methods is designed to obtain three fractions: (i) PCDD/Fs (ii) planar (dioxin-like) PCBs, and (iii) the bulk of PCBs plus PBDEs and PBBs. While the first two fractions (PCDD/Fs and planar PCBs) are generally analysed by gas chromatography coupled to high-resolution mass spectrometry (GC-HRMS) [5], the last can be analysed either by gas chromatography with electron capture detection (GC-ECD) [6] or gas chromatography coupled to low-resolution mass spectrometry (GC-MS) [7]. The main limitation of both instrumental systems is GC coelutions among congeners of the three families (PCBs, PBDEs, and PBBs), which may cause identification 
problems (in ECD detection) and interference problems, increasing the background noise and detection limits (in MS detection). This makes it very difficult to analyse the BFRs, which are usually found at much lower concentrations than PCBs in real samples. Some coelutions detected by ECD can be solved using dual GC columns with different composition and/or characteristics $[9,10]$ or in multidimensional GC (MDGC) systems (i.e. "heart-cutting" MDGC) [11]. The typical coelution of PCB 180 and PBDE 47 can be eliminated using longer GC columns and slower temperature programs than usual [10]. These approaches, however, significantly increase the analysis time. Chlorinated interferences are eliminated by negative ion chemical ionisation (NICI), but this procedure cannot recognise different brominated compounds since only bromine ions can be monitored. Although bromine interferences can be resolved using a GC-MS system in electron impact (EI) ionisation in selective ion monitoring (SIM) mode, chlorinated or brominated compounds with equal $\mathrm{m} / \mathrm{z}$ values cannot be distinguished [12]. HRMS is the most selective and sensitive detection method for these kinds of analysis but acquisition and maintenance costs are very high. The ion trap detector in MS/MS operating mode (ITD(MS/MS)) [7] could be a good option because of its good analytical characteristics and low cost. But, in this case, the analysis of PBDEs in the presence of PCBs, which are normally found at higher concentration than PBDEs in real samples, could increase the background noise and detection limits.

A different strategy to solve coelution problems is prior separation of PCBs from BFR compounds in an additional clean-up step. The different approaches that have been developed, using open liquid chromatography columns with silica gel $[13,14]$ and alumina [15], are not completely satisfactory as sample matrix and analyte concentration can affect the fractionation. In addition, the solid phase extraction (SPE) cartridges usually employed are prepared manually and not very reproducible. This paper describes the development of a high-performance liquid chromatography (HPLC) method using a 2-(1-pyrenyl) ethyldimethylsilylated silica column to achieve satisfactory, reproducible fractionation of PCBs from BFRs (PBDEs and PBBs). The applicability of this method for food analysis was demonstrated by fractionating several food samples.

\section{Experimental}

\subsection{Apparatus}

For HPLC experiments, a HP-1100 Series system (HewlettPackard, Waldbronn, Germany) equipped with an autosampler and variable wavelength detector was used. Instrument control and data acquisition were performed with the HP-1100 Series ChemStation software. Separations were performed on a Cosmosil 5-PYE column, 2-(1-pyrenyl) ethyldimethylsilylated silica gel, $250 \times 4.6 \mathrm{~mm}$ i.d., particle size $5 \mu \mathrm{m}$ from Nacalai Tesque Inc., Promochem GmbH (Kyoto, Japan), and the UVdetector was operated at $225 \mathrm{~nm}$. Different mobile phases, flows, and column temperatures were investigated in order to obtain the best separation between the chlorinated and brominated compounds studied. fifty micro liter was injected for PCB and
PBDE congeners and samples, while $5 \mu \mathrm{L}$ was injected for PBB congeners due to their higher concentration in the commercial standard solution.

Gas chromatography coupled with ion trap mass spectrometry detection in MS/MS or SCAN mode (GC-ITD(MS/MS) or $\mathrm{GC}-\mathrm{ITD}(\mathrm{SCAN})$ ) analysis was performed on a Varian gas chromatograph (CP-3800, CA, USA) equipped with an ion trap detector (ITD, Saturn 2000, Varian). Samples were injected into a programmable temperature vaporising injector (PTV) in hot splitless mode $\left(4 \mu \mathrm{L}\right.$; injection rate: $0.5 \mu \mathrm{L} / \mathrm{s} ; 150{ }^{\circ} \mathrm{C}$, hold for $2 \mathrm{~min}$, and then to $300^{\circ} \mathrm{C}$ at $200^{\circ} \mathrm{C} / \mathrm{min}$; splitless time $2.0 \mathrm{~min}$ ) applying a pressure pulse of 44 psi during $2.10 \mathrm{~min}$. For separation, a low bleed GC capillary column VF-5MS (FactorFour ${ }^{\mathrm{TM}}$, $55 \mathrm{~m}, 0.25 \mathrm{~mm}$ i.d., $0.25 \mu \mathrm{m}$ film thickness) purchased from Varian (CA, USA) was employed. For PBDE and PBB identification the column temperature was programmed as follows: $130^{\circ} \mathrm{C}$ (1 min) to $200^{\circ} \mathrm{C}(1 \mathrm{~min})$ at a rate of $20^{\circ} \mathrm{C} / \mathrm{min}$, then to $280{ }^{\circ} \mathrm{C}$ $(8 \mathrm{~min})$ at $10^{\circ} \mathrm{C} / \mathrm{min}$ and then to $310^{\circ} \mathrm{C}(15 \mathrm{~min})$ at $10^{\circ} \mathrm{C} / \mathrm{min}$. PCBs were identified using the following column temperature program: $100{ }^{\circ} \mathrm{C}(2 \mathrm{~min})$ to $200^{\circ} \mathrm{C}(3 \mathrm{~min})$ at a rate of $30^{\circ} \mathrm{C} / \mathrm{min}$, then to $230^{\circ} \mathrm{C}(15 \mathrm{~min})$ at $3{ }^{\circ} \mathrm{C} / \mathrm{min}$ and then to $270^{\circ} \mathrm{C}(15 \mathrm{~min})$ at $5{ }^{\circ} \mathrm{C} / \mathrm{min}$. Helium was used as carrier gas at a constant flowrate of $1 \mathrm{~mL} / \mathrm{min}$. For the three families, the temperature of the transfer line and the trap were set at $310^{\circ} \mathrm{C}$ and $220^{\circ} \mathrm{C}$, respectively.

\subsection{Reagents and standards}

All solvents employed for the different mobile phases were of HPLC grade. $N$-hexane, $n$-heptane and ethanol were supplied by Scharlau (Barcelona, Spain) and toluene and isooctane were from SDS (Peypin, France). All solvents used for sample preparation and GC-ITD identification were Pestipur quality and were purchased from SDS, except $n$-hexane which was supplied by Merck (Darmstadt, Germany). Sulphuric acid (analysis quality) and silica gel $60(0.063-0.200 \mathrm{~mm})$, used for sample preparation, were obtained from Merck, anhydrous sodium sulphate from J.T. Baker (Deverter, The Netherlands) and Supelclean ${ }^{\mathrm{TM}}$ ENVI $^{\mathrm{TM}}$-Carb SPE cartridges from Supelco (Palo Alto, USA).

Out of 209 possible PCB congeners, for this study we selected PCB \#28, 52, 101, 138, 153, 170, 180, and 194, according to IUPAC numbers [16], since they are relatively abundant in reallife samples [17]. A working stock solution was prepared in $n$-hexane from the commercial PCB standards (Dr. Ehrenstorfer, Augsburg, Germany) containing $1 \mathrm{mg} / \mathrm{L}$ of each native congener.

The ten native PBDE congeners studied (PBDEs \#17, 28, $47,66,85,99,100,153,154$, and 183) were selected on the basis of their relative abundance in commercial mixtures [1]. A working stock solution was prepared in $n$-hexane from the individual PBDE standards (Wellington Laboratories, Ontario, Canada) containing $1 \mathrm{mg} / \mathrm{L}$ of each of ten native compounds.

Commercial mixture Firemaster BP-6 (Promochem, Barcelona, Spain) was used as PBB standard. The major components of this mixture are PBBs \#101, 118, 138, 149, 153, $156,157,167$, and 180 [18]. Firemaster BP-6 was dissolved in $n$-hexane, and a working solution containing $500 \mathrm{mg} / \mathrm{L}$ of the total PBB congeners present in the mixture was prepared. 
Table 1

IUPAC numbers and structures of the PBDE, PCB, and PBB congeners studied in this work

\begin{tabular}{|c|c|}
\hline IUPAC number & Congener structure \\
\hline 47 & $2,2^{\prime}, 4,4^{\prime}$-Tetrabromodiphenyl ether \\
\hline 66 & $2,3^{\prime}, 4,4^{\prime}$-Tetrabromodiphenyl ether \\
\hline 85 & $2,2^{\prime}, 3,4,4^{\prime}$-Pentabromodiphenyl ether \\
\hline 99 & $2,2^{\prime}, 4,4^{\prime}, 5$-Pentabromodiphenyl ether \\
\hline 100 & $2,2^{\prime}, 4,4^{\prime}, 6$-Pentabromodiphenyl ether \\
\hline 153 & $2,2^{\prime}, 4,4^{\prime}, 5,5^{\prime}$-Hexabromodiphenyl ether \\
\hline \multicolumn{2}{|l|}{ Polychlorinated/brominated biphenyls (PCBs/PBBs) } \\
\hline 28 & $2,4,4^{\prime}$-Trichlorobiphenyl \\
\hline 52 & $2,2^{\prime}, 5,5^{\prime}$-Tetrachlorobiphenyl \\
\hline 101 & $2,2^{\prime}, 4,5,5^{\prime}$-Pentachloro/bromobiphenyl \\
\hline 118 & $2,3^{\prime}, 4,4^{\prime}, 5$-Pentabromobiphenyl \\
\hline 138 & $2,2^{\prime}, 3,4,4^{\prime}, 5^{\prime}$-Hexachloro/bromobiphenyl \\
\hline 149 & $2,2^{\prime}, 3,4^{\prime}, 5^{\prime}, 6$-Hexabromobiphenyl \\
\hline 153 & $2,2^{\prime}, 4,4^{\prime} 5,5^{\prime}$-Hexachloro/bromobiphenyl \\
\hline 156 & $2,3,3^{\prime}, 4,4^{\prime}, 5$-Hexabromobiphenyl \\
\hline
\end{tabular}

Table 1 groups the IUPAC numbers and structures for the PBDE, PCB and PBB congeners studied in this work so as to show the degree and location of halogenation in them.

\subsection{Sample preparation}

Different food samples (cheese, milk, and horse mackerel) purchased in several Spanish markets were chosen for application of the fragmentation method investigated. The non-edible part of the food products was removed, and the edible part was stored in stable conditions (freeze-dried) until analysis. Extraction of samples was done by matrix solid phase dispersion (MSPD) as described in detail elsewhere [19]. Between 5 and $10 \mathrm{~g}$ of sample was homogenised with $20 \mathrm{~g}$ of $1: 1$ (w/w) silica gel: anhydrous sodium sulphate. The mixture was ground to a fine powder, loaded into a column and extracted with $400 \mathrm{~mL}$ of 1:1 (v/v) acetone:hexane mixture. Clean-up steps consisted in a multilayer column filled with neutral silica, silica impregnated with sulphuric acid and silica modified with $\mathrm{KOH}$. Both columns were eluted with 125 and $100 \mathrm{~mL}$ of $n$-hexane, respectively. Fractionation between PCDD/Fs and non-ortho PCBs from ortho PCBs plus PBDEs and PBBs was achieved using Supelclean ${ }^{\mathrm{TM}} \mathrm{ENVI}^{\mathrm{TM}}$-Carb SPE cartridges as described elsewhere [6]. The fraction containing ortho PCBs + PBDEs + PBBs was then evaporated under a gentle stream of nitrogen, redissolved with $60 \mu \mathrm{L}$ of $n$-hexane and injected $(50 \mu \mathrm{L})$ into a HPLC for fractionation, using the HPLC method developed for separation of chlorinated and brominated compounds. Both fractions were collected at the end of the HPLC system, evaporated, re-dissolved with $50 \mu \mathrm{L}$ of nonane and injected $(4 \mu \mathrm{L})$ into the GC-ITD(MS/MS) system as described elsewhere $[7,8]$.

\subsection{GC-ITD peak identification}

The GC-ITD(MS/MS) process consists in ionisation of GC effluent by electron impact (EI) at $70 \mathrm{eV}$ followed by isolation of selected parent ions corresponding to each congener and subsequent fractionation of the parent ions by collision-induced dissociation (CID). The most abundant ion was selected from the molecular cluster of each PCB and PBDE as the parent ion for each congener except PBDE 183; in that case the parent ion came from the first EI fragmentation of the molecular ion, 
because its molecular ion exceeded the maximum MS range. Identification by GC-ITD(MS/MS) was based on detection, at the appropriate chromatographic retention time, of the two most abundant ions of the daughter cluster for each congener and the maintenance of the theoretical ratio between them within an appropriate range. The daughter cluster for each congener was the product of the loss of two $\mathrm{Cl}$ fragments by PCBs, and two Br fragments by PBDEs (except for PBDE 183, in which no fragmentation was observed under the experimental conditions). The different parameters affecting MS/MS detection of PCBs and PBDEs have been published elsewhere [7].

To identify PBB congeners, the GC-ITD system was used in the SCAN mode, monitoring ions in the range $500-650 \mathrm{~m} / \mathrm{z}$. Identification was based on the EI spectrum of each congener at $70 \mathrm{eV}$, which has a characteristic isotope cluster of each bromination degree. In addition, it was assumed that the elution order of PBBs on a VF-5MS column is equal to the elution order of PCBs in the same column, and this fact was confirmed for two individual PBB congeners (PBB \#101 and 153). Also, ratios of signal areas of the different PBBs peaks were used for identification taking into account the Firemaster BP-6 composition [18]. In this way the identification of all 9 PBB congeners by GC-EI(MS) was confirmed.

\section{Results and discussion}

\subsection{Development of a HPLC method for fractionation of PCBs from BFRs (PBDEs and PBBs)}

In a previous paper [20], a 2-(1-pyrenyl) ethyldimethylsilylated silica gel column was used for simultaneous separation of coplanar and chiral PCBs based on the number of chlorine atoms in ortho positions, using $n$-hexane at $0.5 \mathrm{~mL} / \mathrm{min}$ and $25^{\circ} \mathrm{C}$. Since these conditions were not appropriate for the separation of PCBs from PBDEs and PBBs, all of which are considered "non-polar" compounds, different chromatographic conditions were assayed. Firstly, increasing percentages $(0,1$, 2 , and $3 \%$ ) of toluene were added to $n$-hexane to increase the elution power of the mobile phase and to decrease the retention time of the most retained congeners of each family. Fig. 1 shows the chromatograms corresponding to PCBs, PBDEs and PBBs for a mobile phase of $n$-hexane (Fig. 1a) and $n$-hexane:toluene $(98: 2, \mathrm{v} / \mathrm{v})$ (Fig. $1 \mathrm{~b}$ ) at $0.5 \mathrm{~mL} / \mathrm{min}$ and $25^{\circ} \mathrm{C}$. Note that the addition of toluene decreased the retention times of the compounds, especially for the last eluting congeners of each family. Although higher percentages than $2 \%$ of toluene in the mobile phase would have been necessary to shorten the retention of the last eluting congeners, there was an important decrease in sensitivity (about six-fold when toluene was increased from 2 to $3 \%$ ) owing to UV-absorption of toluene in the UV region (maximum absorption at $280 \mathrm{~nm}$ ). Ethanol (at 5 and 10\%) was also tested as a modifier in a mobile phase of $n$-hexane, but this polar solvent did not improve on the best separations achieved with toluene. After that, $2 \%(\mathrm{v} / \mathrm{v})$ toluene in $n$-heptane and isooctane was also tested. With isooctane:toluene $(98: 2, \mathrm{v} / \mathrm{v})$, retention times for the PBDE and PBB congeners were longer than for the PCB congeners, favouring separation between the last eluting PCB congeners and the first eluting PBDE and PBB congeners. Using an isooctane:toluene $(98: 2, \mathrm{v} / \mathrm{v})$ mobile phase, a flow-rate of $1 \mathrm{~mL} / \mathrm{min}$ and a column temperature of $45^{\circ} \mathrm{C}$ (the maximum operating temperature of the column was $50^{\circ} \mathrm{C}$ ) were applied to reduce the analysis time.

The last mentioned chromatographic conditions were selected for the fractionation of selected PCB congeners from PBDE and PBB congeners in two mixtures of standard solutions containing respectively PCBs plus PBDEs and PCBs plus PBBs. The chromatograms for both mixtures are shown in Fig. 2, where the time ranges selected for fractionation of the PCBs from the BFR (PBDEs and PBBs) are marked. In the case of $\mathrm{PCB} / \mathrm{PBDE}$ fractionation, the first eluted fraction (F-I) was collected from 4.0 to $5.8 \mathrm{~min}$ and corresponded to almost all PCBs (except PCBs \#170+194), and the second eluted fraction (F-II), from 5.8 to $9.0 \mathrm{~min}$, included majority PBDEs except PBDE 17 and 28. It is important to note that these two PBDEs (PBDEs \#17 and 28) are the smallest components of commercial mixtures of PBDEs and are present in real-life samples in low concentrations [21]. Likewise, one of the PCB congeners (PCB \#194) included in PBDE fraction (F-II) is also a minority congener in
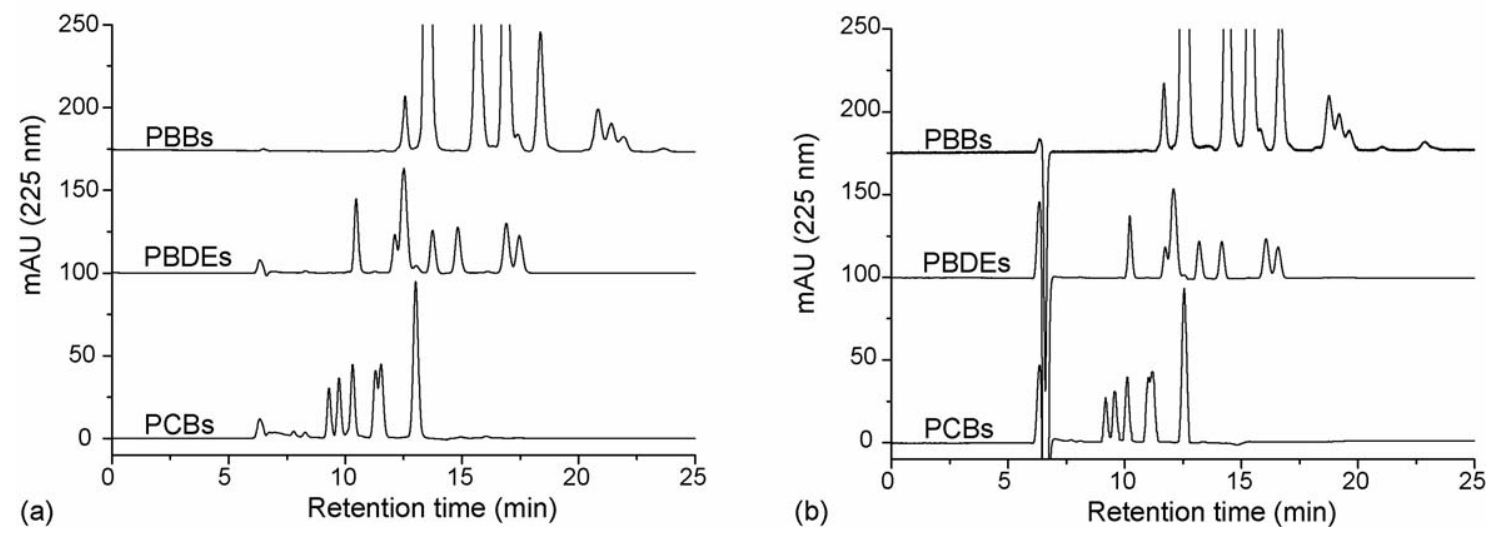

Fig. 1. Chromatograms corresponding to the separation of PCB $(\sim 1 \mathrm{mg} / \mathrm{L}$, each), PBDE $(\sim 1 \mathrm{mg} / \mathrm{L}$, each $)$ and PBB $(\sim 500 \mathrm{mg} / \mathrm{L}$, total $)$ congeners in a $n$-hexane standard solution with a mobile phase of (a) $n$-hexane and (b) $n$-hexane:toluene $\left(98: 2\right.$, v/v). Chromatographic conditions: temperature $25^{\circ} \mathrm{C}$; flow-rate, $0.5 \mathrm{~mL} / \mathrm{min}$ and detection at $225 \mathrm{~nm}$. 

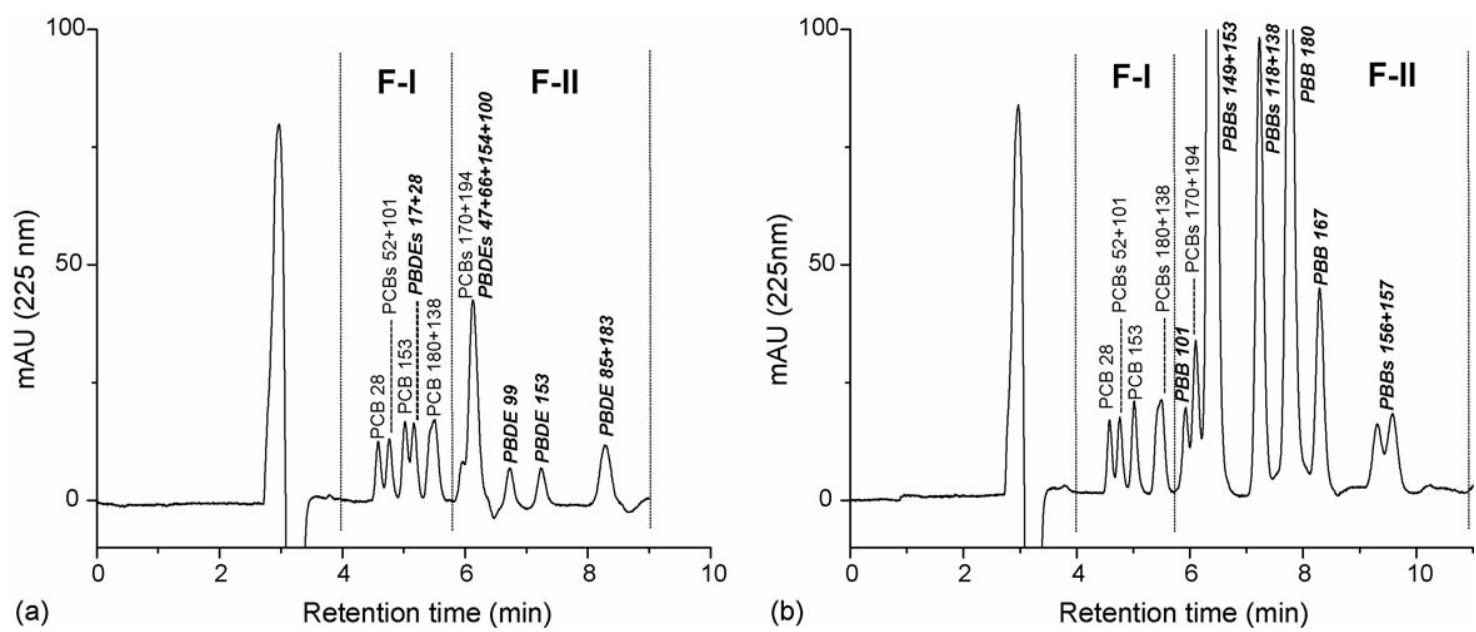

Fig. 2. Chromatograms of mixtures of PCBs with BFRs standard solutions in $n$-hexane containing (a) PCBs ( $\sim 1 \mathrm{mg} / \mathrm{L}$, each) plus PBDEs $(\sim 1 \mathrm{mg} / \mathrm{L}$, each) and (b) PCBs ( $\sim 1 \mathrm{mg} / \mathrm{L}$, each) plus PBBs ( $\sim 500 \mathrm{mg} / \mathrm{L}$, total). Chromatographic conditions: mobile phase, isooctane:toluene $(98: 2, \mathrm{v} / \mathrm{v}) ;$ flow-rate, $1.0 \mathrm{~mL} / \mathrm{min}$, temperature, $45^{\circ} \mathrm{C}$ and detection at $225 \mathrm{~nm}$.

real-life samples [17]. The fractions obtained for PCBs/PBBs improved on PCBs/PBDEs in that only PCBs \#170+ 194 were collected in the PBB fraction and no PBB congeners were present in the PCB fraction. The fractionation achieved separates the bulk of the PCBs for determination of PBDEs (and PBBs), which, as noted earlier, can be affected by the presence of PCB congeners, especially for those congeners present in much higher concentrations than PBDEs (and PBBs) in real samples.

It is important to note that the fractionation power of the proposed method is similar to or even better than those documented in the literature. Planar PCBs were separated from PBDEs when activated carbon and alumina were used in the separation column; nevertheless, non-planar PCBs and PBDEs still appeared in the same fraction, and HRMS was needed to determine PBDEs [15]. The fractionation achieved with silica columns [14,22] was satisfactory for specific PCB and PBDE congeners. For example, Lind et al. [14] studied the separation of the major part of the PCB congeners from PBDEs 47, 99, 100, 153 and 154, but they did not include lower brominated PBDEs, which our method was not able to separate. And again, in the paper published by Martínez et al. [22], quantitative separation between PCBs 28, 52, 118, 138, 153, 180, and 209 and PBDEs 47, 85, 99, 100, 153, and 154 was achieved, but PCBs 170 and 194, which were included in our study (the last two eluting PCB congeners under the selected chromatographic conditions) were not considered.

\subsection{Applicability of the HPLC fractionation method developed for real samples}

After testing of fractionation with standard mixtures, the same conditions were applied to different commercially available food samples. Fig. 3 shows the chromatogram for a standard mixture of PCBs and PBDEs together with the chromatograms of three food samples: cheese, milk and horse mackerel. Note the difference in concentration between PCBs (F-I) and PBDEs
(F-II) in real samples. As was noted in the introduction, PCB concentrations were much higher than PBDE concentrations, so that fractionation produces a PBDE extract free of intereference from the main PCBs. Moreover, the fragmentation achieved under the experimental conditions enabled PCB 180 to be separated from PBDE 47 (see Fig. 3 for peak identification). Both congeners coelute when a GC system is used for their final determination, causing overestimation (mainly for PBDE 47, which is less abundant than PCB 180) of the concentration with common non-selected detectors such as ECD.

Finally, it is important to consider that when real samples were fractionated with the proposed HPLC method, there was an observable improvement in the detection sensitivity achieved by GC-MS. Thus, the limits of detection (LODs, calculated as the concentration corresponding to three times the noise signal) for the three samples studied, with and without the HPLC frac-

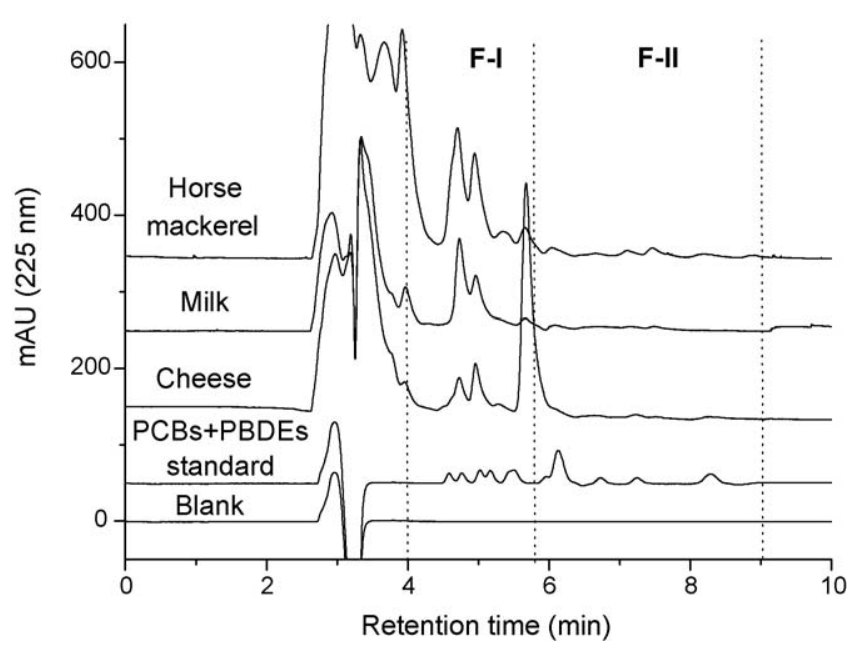

Fig. 3. Chromatogram of cheese, milk, and fish samples including a standard solution in $n$-hexane containing PCBs plus PBDEs ( $\sim 1 \mathrm{mg} / \mathrm{L}$, each). Chromatographic conditions as in Fig. 2. 
Table 2

LODs, expressed in $\mathrm{pg} / \mu \mathrm{L}$ injected, for GC-MS analysis with and without HPLC fractionation

\begin{tabular}{|c|c|c|c|c|c|c|}
\hline \multirow[t]{2}{*}{ PBDE } & \multicolumn{2}{|l|}{ Cheese } & \multicolumn{2}{|l|}{ Milk } & \multicolumn{2}{|l|}{ Horse mackerel } \\
\hline & No fractionation & HPLC fractionation & No fractionation & HPLC fractionation & No fractionation & HPLC fractionation \\
\hline 17 & 1.03 & 0.29 & 0.41 & 0.29 & 0.62 & 0.33 \\
\hline 28 & 1.05 & 0.35 & 0.53 & 0.32 & 0.53 & 0.25 \\
\hline 47 & 0.58 & 0.19 & 0.48 & 0.08 & 0.58 & 0.19 \\
\hline 66 & 0.94 & 0.25 & 0.31 & 0.19 & 0.39 & 0.19 \\
\hline 100 & 0.72 & 0.36 & 0.54 & 0.29 & 0.21 & 0.25 \\
\hline 99 & 1.64 & 0.33 & 1.36 & 0.33 & 1.09 & 0.68 \\
\hline 85 & 2.91 & 1.09 & 1.82 & 1.09 & 2.18 & 1.45 \\
\hline 154 & 0.99 & 0.30 & 0.99 & 0.34 & 0.49 & 0.30 \\
\hline 153 & 1.67 & 0.60 & 1.33 & 0.40 & 1.00 & 0.83 \\
\hline 183 & 1144 & 204 & 817 & 245 & 654 & 245 \\
\hline
\end{tabular}

tionation step prior to GC-MS analysis, are shown in Table 2. Note that LODs for PBDE congeners detected in samples generally decreased when HPLC fractionation was performed prior to GC-MS analysis. These data, then, show the usefulness of the proposed HPLC fractionation method in terms of sensitivity. In addition, this HPLC fractionation method was highly reproducible, given that the R.S.D. (in \%) of elution times for three different injections over 3 months using two different instruments was lower than $0.9 \%$ for all the congeners investigated.

\section{Conclusions}

This paper has described a HPLC method using a column of 2-(1-pyrenyl) ethyldimethylsilylated silica for the fractionation of PCBs from BFRs (PBDEs and PBBs). The method was reproducible (RSD of elution times $<0.9 \%$ ) and enhanced GC-MS sensitivity by making it possible to eliminate interferences from the main PCBs present at high concentrations in real samples. The paper further demonstrates the usefulness of this HPLC fractionation method for the treatment of several food samples prior to a $\mathrm{GC}-\mathrm{MS}$ analysis.

\section{Acknowledgements}

The authors thank the Comunidad Autónoma de Madrid (Spain) for project S-0505/AGR/0312. B. Gómara wishes to thank the University of Alcalá and CSIC for her grants and C. Zuñiga for her kind help. C. García-Ruiz thanks the Ministry of Science and Technology for the Ramón y Cajal program (RYC2003-001).

\section{References}

[1] M. Alaee, P. Arias, A. Sjödin, A. Bergman, Environ. Inter. 29 (2003) 683

[2] A. Covaci, S. Voorspoels, J. de Boer, Environ. Inter. 29 (2003) 735.

[3] Directive 2003/11/CE, 15.2.2003-L42/45.

[4] Council Regulation (EC) 2375/2001, Amending Commission Regulation (EC) 466/2001, Off. J. Eur. Communities 2001, L 321/1-5.

[5] E. Abad, J.J. Llerena, J. Sauló, J. Caixach, J. Rivera, Chemosphere 46 (2002) 1435.

[6] M.A. Concejero, L. Ramos, B. Jiménez, B. Gómara, E. Abad, J. Rivera, M.J. González, J. Chromatogr. A 917 (2001) 227.

[7] B. Gómara, M.A. Fernández, M.J. González, L. Ramos, J. Sep. Sci. 29 (2006) 123.

[8] B. Gómara, L. Herrero, L.R. Bordajandi, M.J. González, Rapid Commun. Mass Spectrom. 20 (2006) 69

[9] M. Alaee, S. Backus, C. Cannon, J. Sep. Sci. 24 (2001) 465.

[10] E. Rogers, M. Petreas, J.-S. Park, G. Zhao, M.J. Charles, J. Chromatogr. B 813 (2004) 269.

[11] L.F. de Alencastro, D. Grandfean, J. Tarradellas, Chimia 57 (2003) 499.

[12] E. Eljarrat, A. de la Cal, D. Barceló, J. Chromatogr. A 1008 (2003) 181.

[13] J. de Boer, Chemosphere 18 (1989) 2131.

[14] Y. Lind, P.O. Darnerud, -S Atuma, M. Aune, W. Becker, R. Bjerselius, S. Cnattingius, A. Glynn, Environ. Res. 93 (2003) 186.

[15] K. D'Silva, A. Fernandes, S. White, M. Rose, Organohalogen Compd. 61 (2003) 179.

[16] K. Ballschmiter, M. Zell, Z. Fresenius, Anal. Chem. 302 (1980) 20.

[17] L. Ramos, E. Eljarrat, L.M. Hernández, J. Rivera, M.J. González, Anal. Chim. Acta 402 (1999) 241.

[18] L.W. Robertson, S.H. Safe, A. Parkinso, E. Pellizari, C. Pochini, M.D. Mullin, J. Agric. Food Chem. 32 (1984) 1107.

[19] L.R. Bordajandi, G. Gómez, M.A. Fernández, E. Abad, J. Rivera, M.J. González, Chemosphere 53 (2003) 163.

[20] L. Ramos, L.M. Hernández, M.J. González, Anal. Chem. 71 (1999) 70.

[21] A. Fernandes, S. White, K. D'Silva, M. Rose, Talanta 63 (2004) 1147.

[22] A. Martínez, M. Ramil, R. Montes, D. Hernanz, E. Rubí, I. Rodríguez, R. Cela Torrijos, J. Chromatogr. A 1072 (2005) 83. 